\title{
ANÁLISE DO CONSUMO ALIMENTAR E DA QUALIDADE DE VIDA DE DOCENTES
}

Fabiana Cristina Gonçalves de Almeida; Caroline Piloto Kuhn; Sandra Cristina Genaro

Universidade do Oeste Paulista - UNOESTE, Curso de Nutrição. Presidente Prudente, SP. E-mail: Fabiana msn cris@hotmail.com

\section{RESUMO}

Este estudo teve como objetivo avaliar o consumo alimentar de 21 professores de nutrição de uma universidade do interior do oeste paulista, e relacionar com sua qualidade de vida. Foram utilizados o Questionário de Frequência de Consumo Alimentar e a versão brasileira do Questionário de Qualidade de Vida SF-36. Os dados foram submetidos à análise estatística descritiva e apresentados em tabelas na forma de frequência absoluta e percentual. Utilizou-se o coeficiente de correlação de postos de Spearman pela quantidade de informações obtidas. As análises estatísticas foram realizadas no programa SPSS 21.0, sendo considerado como estatisticamente significante quando a probabilidade for menor ou igual a 0,05 (p-valor $<0,05$ ). Os resultados apontaram baixo consumo de leite e derivados, leguminosas, cereais integrais, frutas, legumes, verduras, com uma ingestão de carne vermelha excessiva e o consumo de alimentos industrializados superior ao esperado em conjunto com a falta de atividade física regular.

Palavras chave: Alimentação. Saúde. Professor. Atividade Física. Doenças Crônicas.

\section{ANALYSIS OF FOOD CONSUMPTION AND TEACHERS QUALITY OF LIFE}

\begin{abstract}
This study aimed to evaluate the food intake of 21 teachers of nutrition at a university in the interior of São Paulo west, and relate to their quality of life. the Food Consumption Frequency Questionnaire and the Brazilian version of the Quality of Life Questionnaire SF-36 were used. The data were submitted to descriptive statistics and presented in tables in the form of absolute and percentage frequency. We used the Spearman's rank correlation coefficient for the amount of information obtained. Statistical analyzes were performed using SPSS 21.0, and considered statistically significant when the probability is less than or equal to $0.05(p<0.05)$. The results showed low consumption of dairy products, legumes, whole grains, fruits, vegetables, with an intake of excessive red meat and the consumption of processed foods than expected together with the lack of regular physical activity.
\end{abstract}

Key words:

Feeding. Health. Teacher. Physical activity. Chronic diseases. 


\section{INTRODUÇÃO}

Profissionais que acumulam excesso de trabalho, muitas vezes com três jornadas e horários irregulares, podem desencadear um processo de estresse que repercute em sua saúde (BRAGA, PATERNEZ, 2011; OLIVEIRA et al., 2012). Esta rotina pode refletir em sua alimentação, propiciando maus hábitos alimentares (BRAGA, PATERNEZ, 2011).

Os professores formam uma categoria profissional exposta a grandes riscos psicossociais, devido à difícil organização escolar e por se depararem diariamente com situações que desequilibram suas expectativas e causam esgotamento mental (PEREIRA, TEIXEIRA, LOPES, 2013).

Este estudo teve como objetivo avaliar o consumo alimentar de professores do curso de nutrição de uma universidade do interior do oeste paulista, e relacionar com sua qualidade de vida.

\section{METODOLOGIA}

Este estudo caracteriza-se por ser do tipo observacional, descritivo com delineamento transversal de análise quali-quantitativa realizado no Bloco B da Universidade do Oeste Paulista, durante os meses de maio a junho.

A pesquisa foi iniciada após a aprovação do Comitê de Ética em Pesquisa da Universidade do Oeste Paulista (UNOESTE), com o número do protocolo 3060. Os sujeitos que concordaram em participar da pesquisa assinaram um termo de consentimento livre e esclarecido (TCLE).

A população alvo foi composta por 48 professores pertencentes ao curso de Nutrição, porém somente 21 entregaram os questionários no prazo estipulado (15 dias após sua posse).

Foram incluídos no estudo, docentes do curso de nutrição de ambos os sexos, sem distinção de idade ou raça; e excluído os professores que não pertenciam à unidade acadêmica selecionada e que não eram parte do curso mencionado.

Como protocolo de avaliação, foram utilizados questionários autoaplicáveis, composto por Anamnese elaborado pelas próprias pesquisadoras, com a finalidade de investigar presença de doenças existentes; Questionário de frequência de consumo alimentar - QFA (RIBEIRO et al, 2006); versão brasileira do questionário de qualidade de vida SF-36 (CICONELLI, 1999).

Os dados foram submetidos à análise estatística descritiva e apresentados em tabelas na forma de frequência absoluta e percentual. Utilizou-se o coeficiente de correlação de postos de Spearman pela quantidade de informações obtidas. As análises estatísticas foram realizadas no programa SPSS 21.0, sendo considerado como estatisticamente significante quando a probabilidade for menor ou igual a 0,05 ( $p$-valor $<0,05$ ).

\section{RESULTADOS}

Dos professores que responderam o questionário, a maioria $(76,2 \%)$ era do sexo feminino, como demonstrado no Gráfico 1.

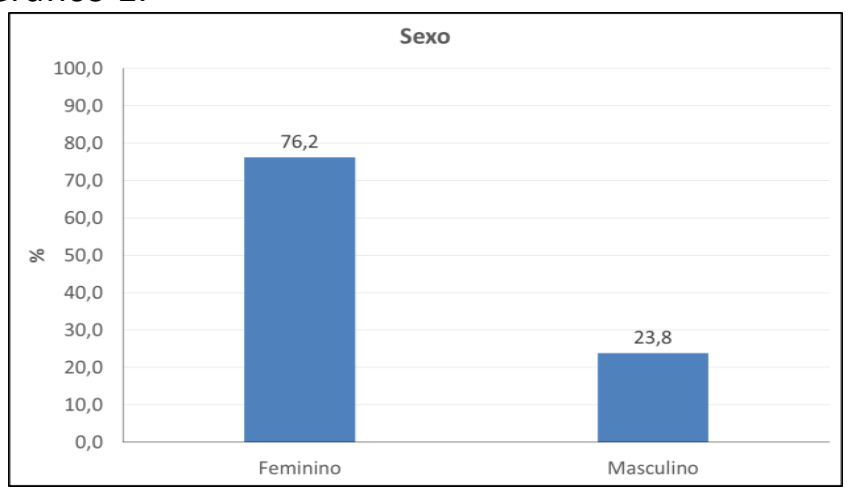

Gráfico 01. Porcentagem de mulheres e homens na pesquisa 
O Gráfico 2 demonstra as doenças que os professores já possuíam, o qual a hipertensão foi a mais prevalente, com $23,8 \%$ dos casos.

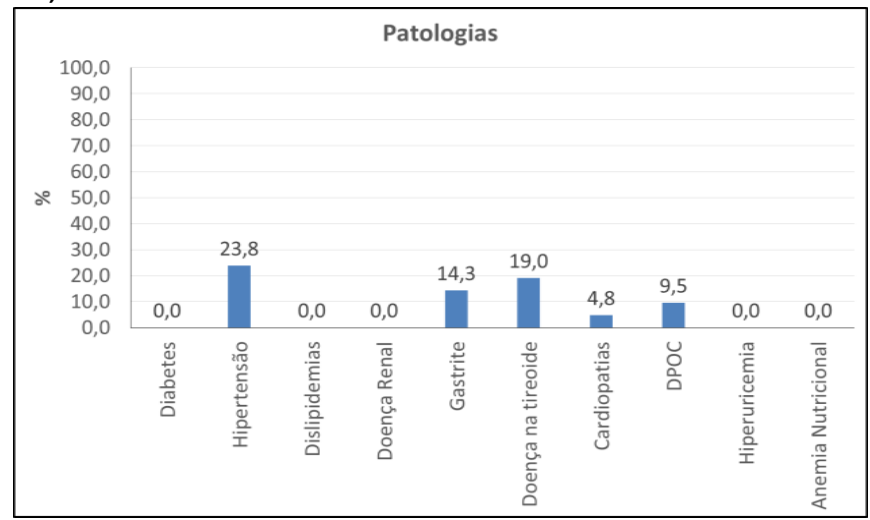

Gráfico 02. Doenças apresentadas pelos professores

O Gráfico 3 demonstra o hábito intestinal dos professores do estudo.

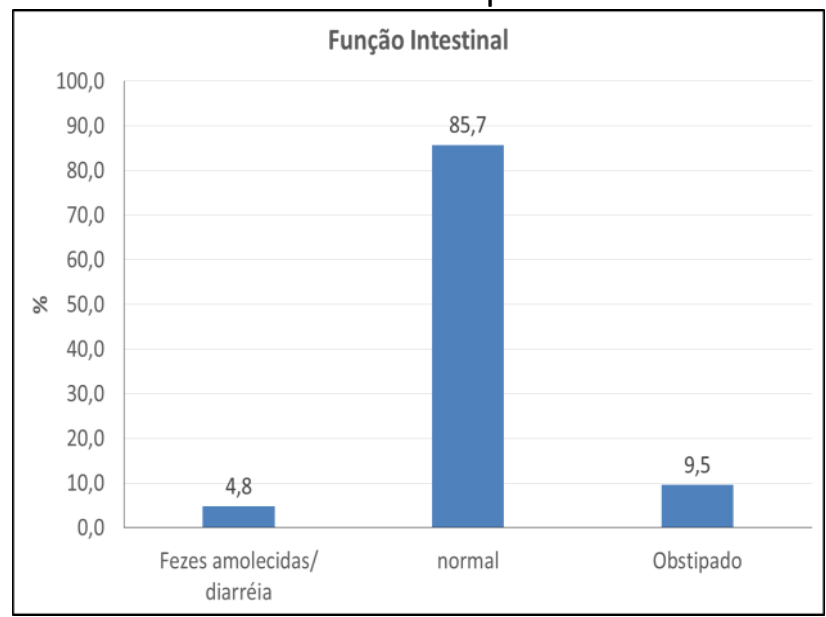

Gráfico 03. Hábito intestinal

O Gráfico 4 apresenta características dos hábitos gerais dos professores do estudo, no qual a maioria $(61,9 \%)$ relatou ingerir bebida alcoólica eventualmente.

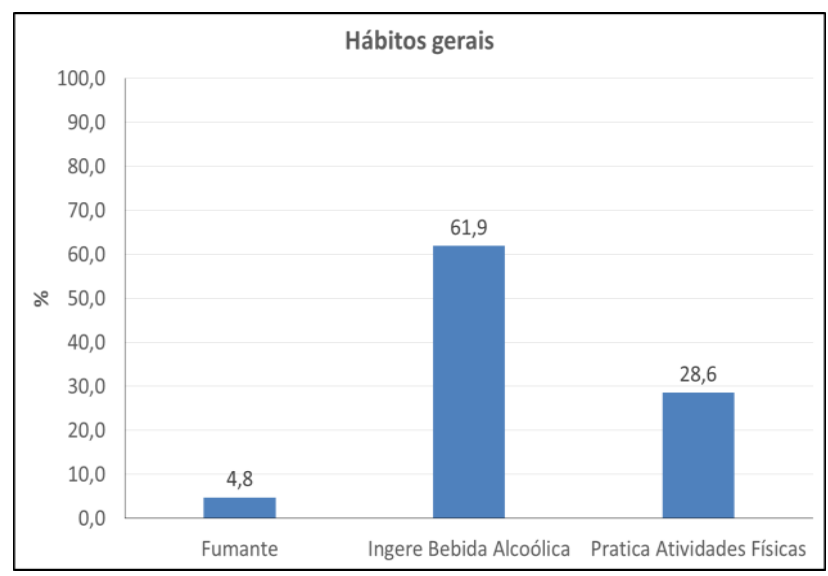

Gráfico 04. Hábitos gerais

Com relação à qualidade de vida, mais da metade dos entrevistados $(52,4 \%)$ acredita ter uma boa saúde, sem dificuldades para realizarem tarefas habituais; ou que seus problemas emocionais são incapazes de interferir em suas atividades sociais habituais, como observado no Gráfico 5. 


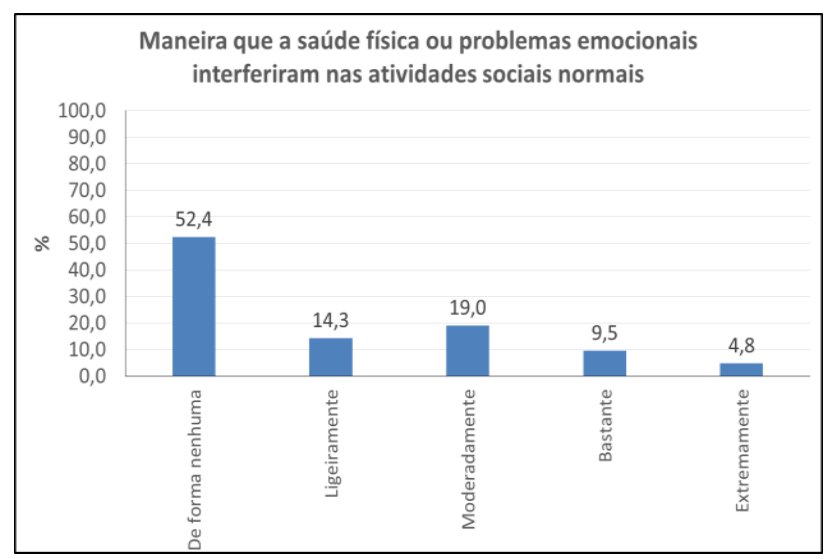

Gráfico 05. Maneira que a saúde física ou problemas emocionais interferem nas atividades sociais normais

Na Tabela 1 é apresentado o p-valor do Coeficiente de Correlação de Spearman entre a variável qualidade da alimentação e as componentes da qualidade de vida. Pelos resultados obtidos não há evidências que haja alguma relação entre a qualidade da alimentação e qualquer um dos componentes da qualidade de vida para os professores participantes do estudo.

Tabela 01. Estatísticas dos componentes do instrumento de medição de qualidade de vida e qualidade da alimentação.

\begin{tabular}{lllllll}
\hline \multirow{2}{*}{ Componentes } & \multicolumn{2}{l}{ Estatísticas } & & & \\
\cline { 2 - 5 } & $\mathbf{N}$ & Média & $\begin{array}{l}\text { Desvio } \\
\text { padrão }\end{array}$ & Mínimo & Máximo & p-valor \\
\hline Qualidade da alimentação & 21 & 66,5 & 9,6 & 49,5 & 81,9 & - \\
\hline Saúde física & & & & & & \\
$\quad$ Capacidade funcional & 21 & 80,7 & 18,3 & 45,0 & 100,0 & 0,559 \\
$\quad$ Aspectos físicos & 21 & 82,1 & 32,7 & 0,0 & 100,0 & 0,613 \\
$\quad$ Dor & 21 & 70,2 & 28,5 & 22,0 & 100,0 & 0,652 \\
$\quad$ Estado geral saúde & 21 & 66,5 & 20,3 & 20,0 & 100,0 & 0,958 \\
\hline Saúde mental & & & & & & \\
$\quad$ Aspectos emocionais & 21 & 74,6 & 36,4 & 0,0 & 100,0 & 0,305 \\
$\quad$ Aspectos sociais & 21 & 76,8 & 28,3 & 0,0 & 100,0 & 0,227 \\
$\quad$ Vitalidade & 21 & 54,5 & 24,5 & 10,0 & 100,0 & 0,409 \\
$\quad$ Saúde mental & 21 & 69,9 & 23,0 & 20,0 & 100,0 & 0,955 \\
\hline
\end{tabular}

A característica alimentar dos professores foi observada no Gráfico 6, onde verificou-se a qualidade da alimentação, classificada em três níveis de pontuação: Bom, Regular e Ruim. 


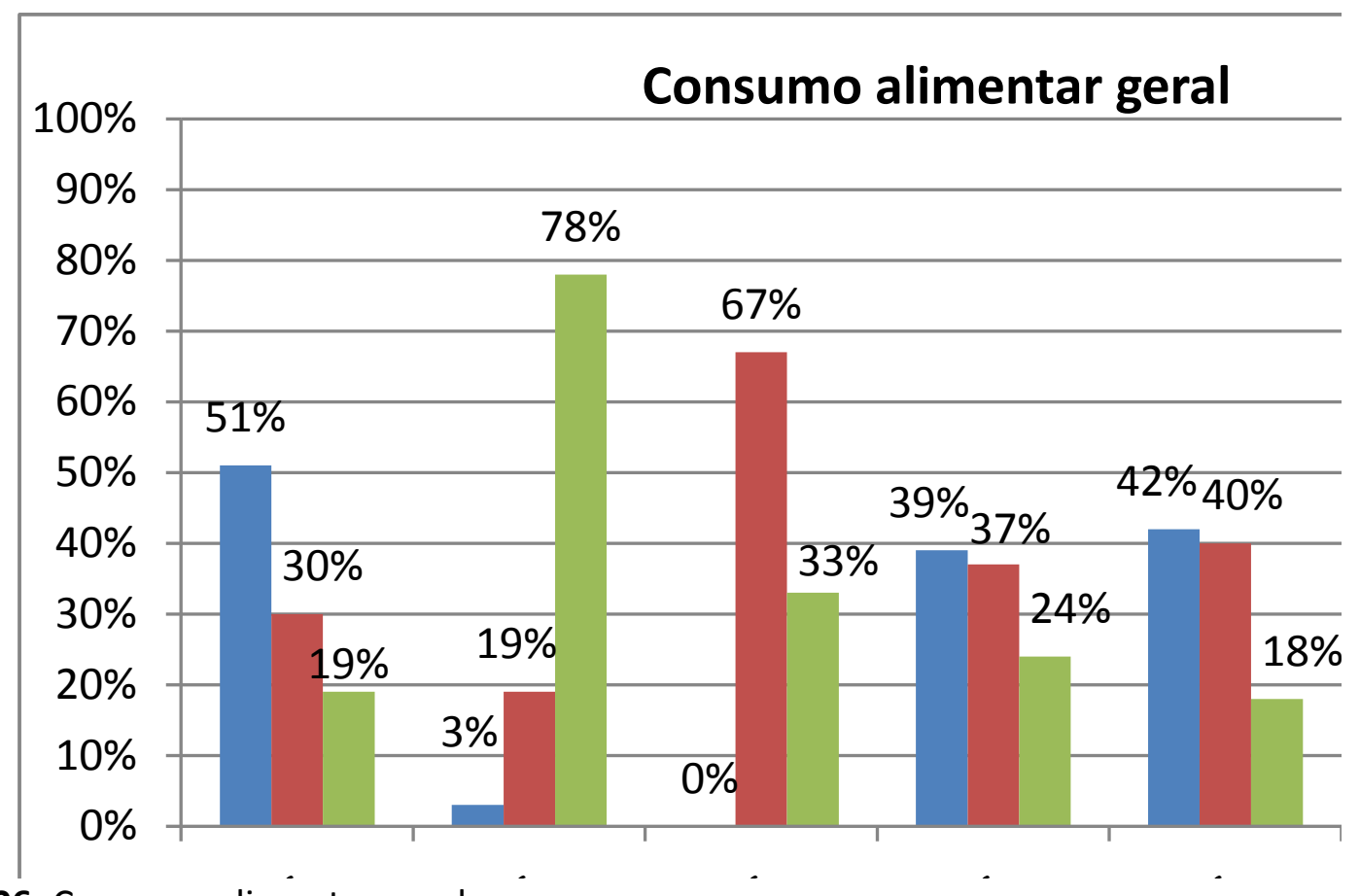

Gráfico 06. Consumo alimentar geral

\section{DISCUSSÃO}

As doenças apresentadas pelos professores, no presente estudo, em destaque a hipertensão, podem ter sido causadas por vários fatores, dentre eles o estilo de vida e a má alimentação, incluindo alimentos processados com excesso de sódio, condimentos, frituras, fast foods, dentre outros (BERNAUD et al., 2013).

A má alimentação, juntamente com o estilo de vida e o estresse do cotidiano pode refletir também no hábito intestinal, causando obstipação pela ausência do consumo de fibras na alimentação e também o aparecimento de doenças como síndrome do cólon irritável que altera obstipação com diarreia (QUIGLEY et al., 2009).

Mesmo a maioria dos professores demonstrando percepção positiva sobre sua qualidade de vida e saúde, notou-se uma qualidade alimentar deficiente em vários nutrientes quando observado o consumo de leite e derivados; cereais integrais; leguminosas; frutas, verduras e legumes. Além disso, a maioria relatou consumir uma maior quantidade de carne vermelha (bovina), sendo favorável a um maior risco de doenças cardiovasculares e câncer de colorretal, de acordo com SCHUSTER, OLIVEIRA, DAL BOSCO (2015) e ZANDONAI, SONOBE, SAWADA (2011). Ainda de acordo com a qualidade alimentar avaliada, o relato de consumo de alimentos industrializados foi elevado comparado com os alimentos minimamente processados. Dessa forma, todos esses fatores colaboram com o aparecimento de doenças crônicas.

\section{CONCLUSÃO}

Por se tratar de docentes da área da saúde especificamente do curso de nutrição, esperava-se uma melhor qualidade alimentar. Talvez pela falta de tempo, a opção pela forma mais prática de alimentação (alimentos industrializados) é maior; deixando por vezes de consumir uma variedade de alimentos frescos minimamente processados, com melhor qualidade em relação à diversidade de nutrientes.

Uma alimentação saudável com a prática regular de atividade física é capaz de evitar vários problemas de saúde, inclusive doenças crônicas como diabetes, hipertensão, dislipidemias, doenças cardiovasculares, obesidade e até alguns tipos de câncer. 
Com base nesses resultados, sugere-se criação de novas opções para alimentação saudável próximo ao local de trabalho.

\section{REFERÊNCIAS}

BRAGA, M. M; PATERNEZ, A. C. A. C. Avaliação do consumo alimentar de professores de uma universidade particular da cidade de São Paulo (SP). Revista Simbio-logias, Botucatu, v. 4, n.6, p. 84-97, dez. 2011. Disponível em:

<http://www.ibb.unesp.br/Home/Departamentos/Educacao/SimbioLogias/AvaliacaodoConsumoA limentardeProfessoresdeUmaUniversidadeParticulardaCidadedeSaoPau.pdf $>$. Acesso em 18 jul. 2015.

BERNAUD, F.S. R, et.al. Fibra alimentar - Ingestão adequada e efeitos sobre a saúde do metabolismo. Arq. Bras. Endocrinol. Metab; p397-402, 2013.Disponível em: http://www.scielo.br/pdf/abem/v57n6/01.pdf. Acesso em: 22/06/2016.

CICONELLI, R. M., et al. Tradução para língua portuguesa e validação do questionário genérico de avaliação de qualidade de vida SF-36 (Brasil SF-36). Rev Bras Reumato. v.3, n.39, p.143-50, 1999. Coeficiente de correlação de postos de Spearman. Disponível em leg.ufpr.br/ silvia/CE701/node80.html. Acessado em 30/05/2016.

OLIVEIRA, et al. Gênero e qualidade de vida percebida - estudo com professores da área de saúde. Revista Ciência \& Saúde Coletiva. Rio de Janeiro-RJ, v.17, n.3, p.742, março, 2012.

PEREIRA, E.F; TEIXEIRA, C.F; LOPES, A.S. Qualidade de vida de professores de educação básica do município de Florianópolis SC, Brasil. Revista Ciência \& Saúde Coletiva. Rio de Janeiro-RJ, v.18, n.7, julho 2013.

QUIGLEY, E. Síndrome do intestino irritável, uma perspectiva mundial. World Gastroenterology Organisation Global Guidelines, p.1-23, 04/2009.

SCHUSTER J, et al. O Papel da Nutrição na Prevenção e no Tratamento de Doenças Cardiovasculares e Metabólicas. Revista da Sociedade de Cardiologia do Estado do Rio Grande do Sul, n 28, pg 1-6, 2015. Disponível em:

file://E:/Users/Dudu/Desktop/concluindo\%20tcc/consumo\%20de\%20carnes\%20e\%20doen\%C3\% A7as\%20cardiovasculares.pdf. Acesso em: 20/06/2016.

ZANDONAI. A.P; et al. Os fatores de riscos alimentares para câncer colorretal relacionado ao consumo de carnes. Revista Escola de Enfermagem USP. Ribeirão Preto SP,vol.46, n.1, pg 234239, 2012. Disponível em : http://www.scielo.br/pdf/reeusp/v46n1/v46n1a31.pdf. Acesso em: 20/06/2016. 